\title{
A study of sorption phenomena on steel surface in solutions of aminotris (methylenephosphonic acid)
}

\author{
A.N. Serov, ${ }^{1 *}$ N.S. Grigoryan, ${ }^{1}$ (D) V.S. Makhina, ${ }^{1}$ T.A. Vagramyan, ${ }^{1}$ (D) \\ A.A. Abrashov, ${ }^{1}$ V.E. Kasatkin ${ }^{2}$ and I.A. Arkhipushkin ${ }^{2}$ \\ ${ }^{1}$ Federal State Budgetary Educational Institution of Higher Education \\ “D.I. Mendeleev Russian Chemical-Technological University”, Miusskaya square, 9, \\ 125047 Moscow, Russian Federation \\ ${ }^{2}$ A.N. Frumkin Institute of Physical Chemistry and Electrochemistry, Russian \\ Academy of Sciences, Leninsky prosp, 31, 119071 Moscow, Russian Federation \\ *E-mail:serov@muctr.ru
}

\begin{abstract}
The behavior of carbon steel in solutions containing components of alkaline cyanide-free electrolyte for bronzing has been studied. It has been shown by the open circuit potential measurement method that contact deposition of copper on steel surface is thermodynamically possible in the solutions studied. Impedance spectrometry data show that the interaction of steel with the components of the copper-containing alkaline solution leads to the formation of a passivating film on its surface, which inhibits contact copper deposition. It has been shown that the formation of a passivation film is also possible if no copper is present in the solution. The process is significantly accelerated in solutions containing divalent copper ions due to the interaction of iron corrosion products with the divalent copper - aminotris(methylenephosphonic acid) complex by the electrophilic substitution mechanism. This interaction results in the deposition of insoluble copper hydroxides on the steel surface. Data of X-ray photoemission analysis confirm that the film contains compounds of mono- and divalent copper, as well as ligand molecules.

Received: June 7, 2021. Published: July 14, 2021

doi: $\underline{10.17675 / 2305-6894-2021-10-3-7}$
\end{abstract}

Keywords: contact deposition of copper, alkaline cyanide-free bronzing, impedance spectrometry.

\section{Introduction}

When copper or copper-containing galvanic coatings are deposited onto a steel base, there is a risk of contact copper deposition after immersing an item in the electrolyte but before cathodic polarization is applied. Copper deposited in contact manner adversely affects the adhesion of the coating deposited later by impairing its adhesion to the steel base. Moreover, contact exchange results in contamination of the electrolyte with iron ions that can adversely affect the performance of the electrolyte, especially in case of the electrodeposition of alloys. 
Various methods or their combinations are used to inhibit this process, including:

- Preliminary coating (e.g., with nickel);

- Use of inhibiting additives that provide steel protection in contact with a solution without cathodic polarization and hinder copper reduction;

- Loading steel parts into the electrolyte "under current", i.e., under cathodic polarization;

- Use of electrolytes in which the copper ion is bound into a strong complex that prevents contact deposition.

The most popular method to solve the issue of contact exchange in electrolytes for deposition of copper and its alloys involves binding copper ions into complex compounds [1-3]. At the same time, practical recommendations for the operation of alkaline cyanidefree copper plating electrolytes (pyrophosphate, ethylenediamine) that are most common in the industry, such as loading parts into the electrolyte under current, indicate that these complexes are not strong enough to prevent the contact deposition of copper on a steel surface [4]. According to literature data, the stability constants of organophosphate copper complexes are comparable to the stability constant of the pyrophosphate copper complex [5].

In the development of an alkaline cyanide-free electrolyte for bronzing based on aminotris (methylenephosphonic acid), it was noted that in the absence of external polarization, the formation of contact copper is not observed on the surface of a steel electrode even after prolonged exposure of steel to the electrolyte, though, in accordance with literature data on instability constants, its formation is possible. Thus, it can be assumed that the inhibition of the contact exchange reaction is due not only and not so much to the binding of copper into a complex ion but also to the adsorption processes on the steel surface involving the organophosphorus ligand.

This work deals with a study on the mechanism of inhibition of the contact reduction of copper on the surface of steel in solutions containing components of cyanide-free electrolyte bronzing.

\section{Experimental}

A sample of 08ps carbon steel with an area of $1 \mathrm{~cm}^{2}$ was used as the working electrode. Just before the experiment, a steel sample was ground with R-1000 abrasive paper and polished with GOI paste, then degreased with Viennese lime and activated in 5\% sulfuric acid solution. After each surface preparation operation, the sample was washed with distilled water.

Solutions were made of "chemically pure" grade reagents. The compositions of the model solutions studied are given in Table 1. 
Table 1. Compositions of the model solutions.

Solution 1

Aminotris(methylenephosphonic acid) (ATMP)

$\mathrm{CuSO}_{4} \cdot 5 \mathrm{H}_{2} \mathrm{O}$
Solution 2

$0.25 \mathrm{M}$
$0.25 \mathrm{M}$

$0.11 \mathrm{M}$

Experiments were carried out at solution temperatures of 20 and $55^{\circ} \mathrm{C}$. The measurements were carried out in solutions without stirring or with stirring with a magnetic stirrer at $700 \mathrm{rpm}$.

Polarization and impedance measurements were performed using an IPC-Pro MF potentiostatic complex with an FRA-2 unit (Russia). The measurements were carried out using a temperature-controlled three-electrode cell with a volume of $250 \mathrm{ml}$. A silver chloride electrode was used as the reference electrode, and a platinum anode with an area of $3 \mathrm{~cm}^{2}$ served as the auxiliary electrode.

To measure the open circuit potential of the electrode, we used an IPC-Recorder (Russia) that allows one to record the potential variation over time. The measurements were carried out for at least 5 minutes until a constant potential was attained.

The frequency dependences of the impedance were obtained in potentiostatic mode at the open circuit potentials, with superposition of a harmonic signal with an amplitude of $10 \mathrm{mV}$ in the frequency range of $20 \mathrm{kHz}-0.13 \mathrm{~Hz}$. The impedance spectra obtained were processed and equivalent circuits that adequately reflect the experimental plots were simulated using the ZMonitor and DCS programs ${ }^{1}$. Each measurement was carried out at least in duplicate.

\section{Results and Discussion}

To estimate the behavior of steel in solutions of the organophosphorus compound, the open circuit electrode potentials were measured (Figure 1). One can see that in the ligand solution, i.e., solution 1 (Figure 1A, curve 1) in the absence of copper ions, the potential of a steel electrode is shifted by $200 \mathrm{mV}$ in the negative direction compared to the background solution (dashed line in Figure 1A). This shift may be due to the formation of strong complexes of iron ions with the ligand (ATMP). It should be noted that stirring the solution results in an even greater negative shift of the electrode potential (by $300 \mathrm{mV}$ ) (Figure 1A, curve 1'). This fact may be due to the diffusion restrictions with respect to the ligand and/or oxidant (oxygen) in the boundary region that is enriched with iron ions in the course of corrosion.

\footnotetext{
${ }^{1}$ The ZMonitor and Dummy Circuits Solver programs were designed by V.E. Kasatkin specifically for use in combination with the IPC-FRA electrochemical complex.
} 
It should be noted that heating the solution also shifts the open circuit potential of a steel electrode in the ligand solution by $300 \mathrm{mV}$ in the negative direction, which indirectly confirms the assumption made above.

Along with this, it was found that stirring insignificantly affects the potential shift in the hot solution (curves 2 and $2^{\prime}$ ), probably due to the complete binding of iron ions into complex compounds.

The addition of copper ions to a solution has a significant effect on the potential of steel electrode (Figure 1B). One can see that if copper ions are present in the solution, the potential is significantly shifted to the positive region as compared to the solution without copper under all the conditions studied, but it still remains more negative than the potential of a copper electrode in this solution (dashed line in Figure 1B).

This potential shift in the presence of copper ions in the solution could be explained by the appearance of a second oxidizer (copper ions) in the solution, which intensifies the iron dissolution process. It is known from the literature that dissolving iron can displace copper from the complex ion by the electrophilic substitution mechanism [6]. However, no contact reduction of copper on the steel surface was observed visually.

These results may indicate that the observed potential shift is associated with the formation of passivating adsorption films that may comprise mono- and divalent copper compounds, iron compounds, and ligand (ATMP) molecules. As the temperature rises, the potential shifts more strongly to the positive region, which confirms the above assumption. An increase in the iron dissolution rate apparently leads to an increase in the rate of copper ion replacement by an iron ion in the complex. This, in turn, accelerates the formation of a passivating film on the electrode surface. Stirring practically does not affect the potential, both in cold and hot solutions, which is explained by the absence of diffusion restrictions on copper ions in the solution containing $0.11 \mathrm{~mol} / \mathrm{L} \mathrm{Cu}^{2+}$.

The electrochemical impedance spectroscopy method is widely used to study the interface processes in electrochemical systems. The advantage of this method is not only that it allows one to estimate the corrosion resistance of a metal under the effect of various factors but also that information about the mechanism of reactions occurring on the metal being studied can be obtained.

In impedance spectroscopy, the impedance modulus measured at the lowest frequency $(0.13 \mathrm{~Hz}$ in this work) is the closest to the polarization resistance under constant current polarization. In other words, the wider the hodograph loop along the real axis (i.e., the higher the resistance along the real axis that corresponds to its right low-frequency end), the lower the rate of corrosion processes occurring on the steel electrode surface. It is known from theory that the Nyquist diagram (impedance hodograph) of a capacitor and resistor connected in parallel takes the shape of a regular semicircle and this diagram simulates the process of charge transfer across a phase interface. If the capacitance $C$ in this scheme is replaced by a constant phase element $\mathrm{CPE}$, it becomes possible to simulate hodographs with a flattened arc that correspond to an imperfect capacitor with a diffuse structure of the ionic plate. 

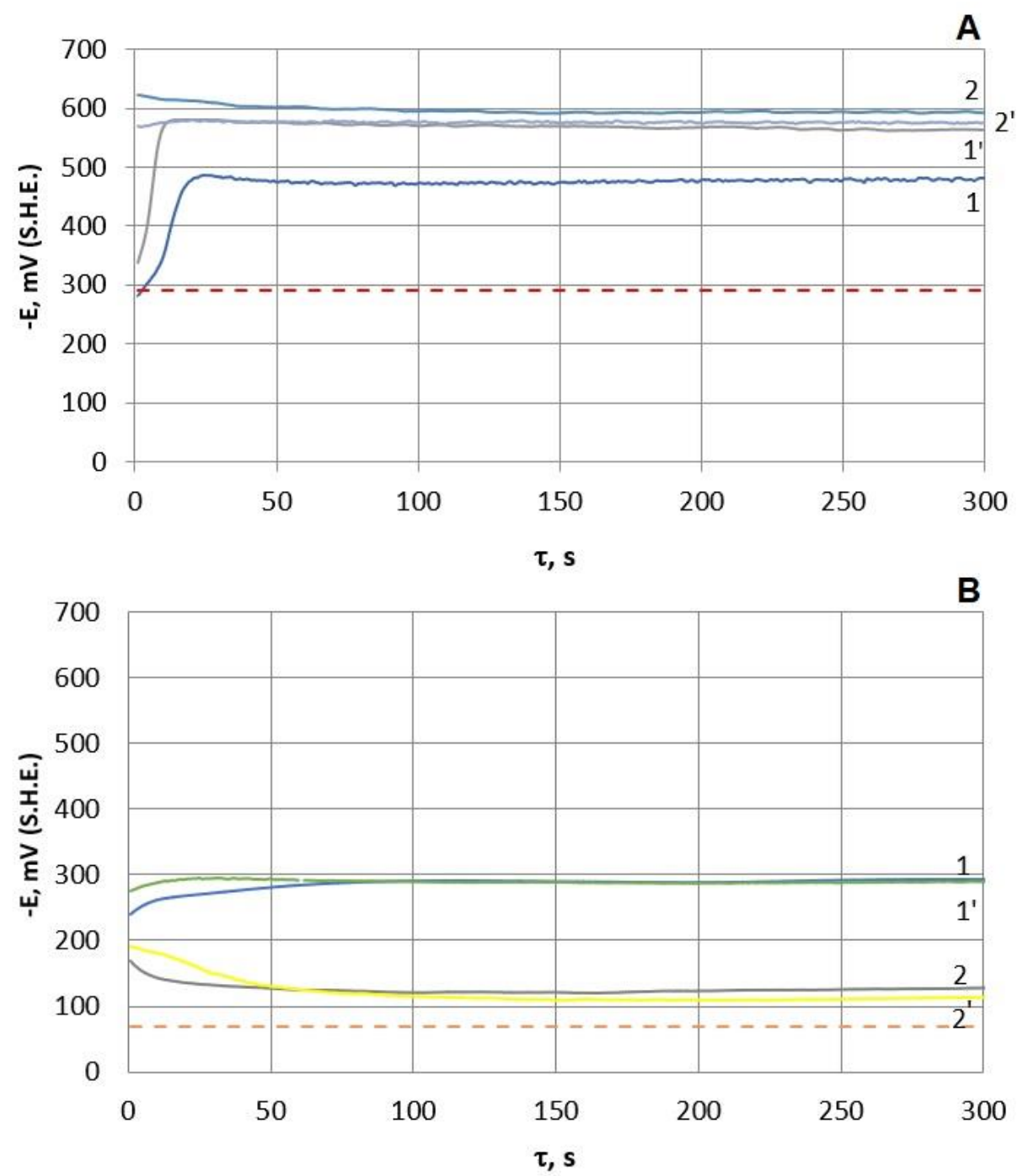

Figure 1. Chronopotentiograms of steel electrode. A - solution 1; B - solution $2 ; 1,1^{\prime}-20^{\circ} \mathrm{C}$; $2,2^{\prime}-55^{\circ} \mathrm{C} ; 1,2$ - without stirring; $1^{\prime}, 2^{\prime}-$ with stirring.

Figure 2 shows the Nyquist diagrams obtained in the solutions studied. One can see that the hodographs obtained in a solution containing no copper ions (Figure 2A) differ in behavior more strongly than it could be expected from the results of measuring the open circuit potential.

The lowest rate of corrosion processes and the greatest hindrance of charge transfer across the interface are observed in the cold solution in the absence of stirring (Figure 2A, curve 1). In combination with the data on the open circuit potentials of the steel electrode, this may indicate that iron dissolves rather intensely (as indicated by the maximum displacement of the potential to the positive region), but without mixing iron ions passing into the solution remain in the near-surface layer, thus hindering the further charge transfer through the double layer.

According to the Pourbaix diagrams (Figure 2), at about $\mathrm{pH} 10$ iron dissolves either to give the $\mathrm{Fe}^{2+}$ ion (Figure $2 \mathrm{~A}$ ) or the $\mathrm{FeOH}^{+}$ion (Figure $2 \mathrm{~B}$ ). 

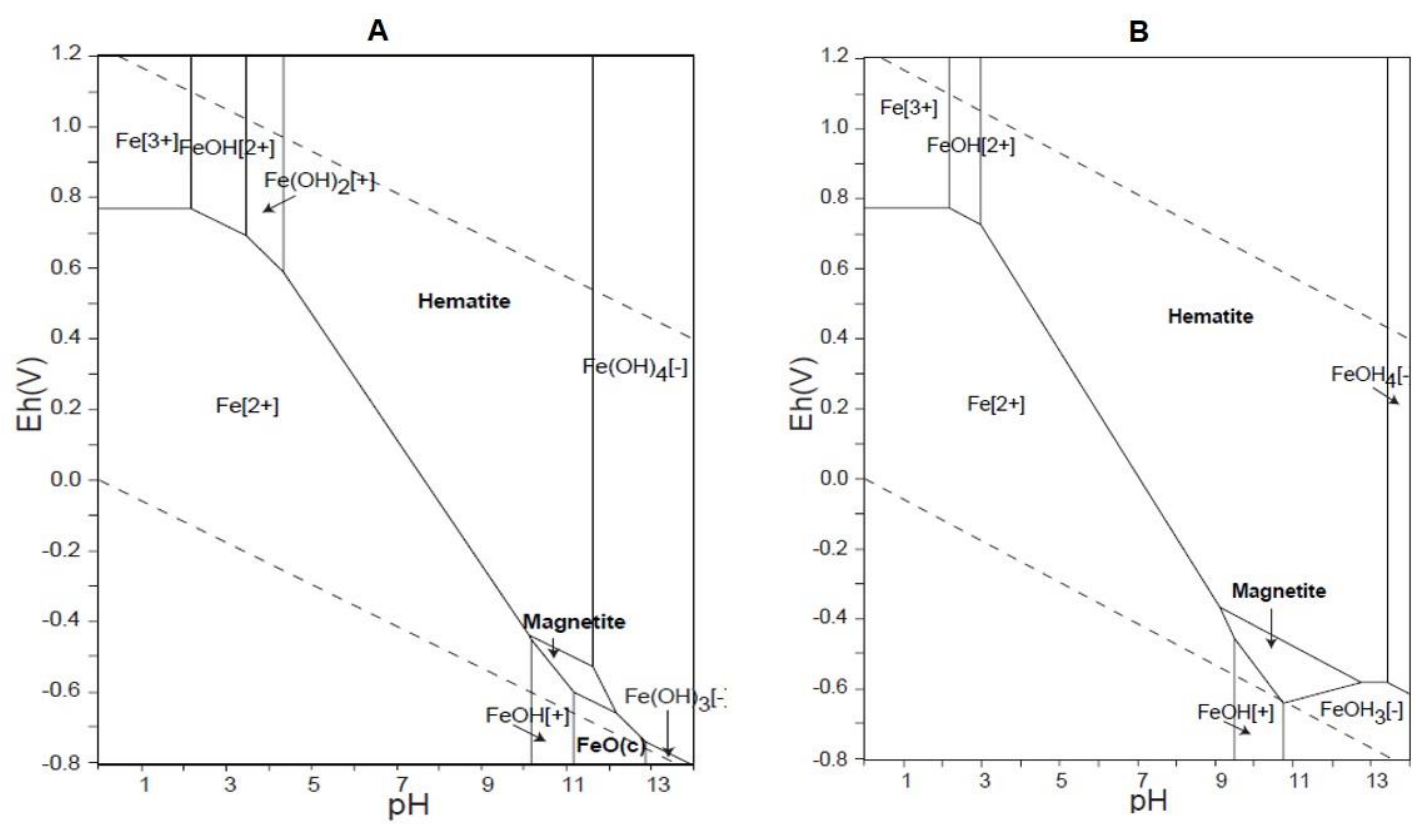

Figure 2. Pourbaix diagrams of the $\mathrm{Fe}-\mathrm{O}-\mathrm{H}$ system.

Under conditions of corrosion with oxygen depolarization in an alkaline medium, the near-electrode layer can be alkalized by the reaction

$$
\mathrm{O}_{2}+2 \mathrm{H}_{2} \mathrm{O}+4 \mathrm{e} \rightarrow 4 \mathrm{OH}^{-}
$$

Alkalization can give rise to the formation of iron hydroxide films on a steel surface. The formation of a new non-conductive phase on the electrode surface causes an increase in the polarization resistance in the low-frequency part of the plot, which is consistent with the appearance of a second semicircle on the hodograph in the low-frequency region (Figure 3A, curve 1).

In accordance with the assumption that the charge transfer is hindered due to the accumulation of steel corrosion products in the near-electrode layer, stirring results in an increase in the charge transfer rate, as the decrease in the hodograph radius indicates (Figure $3 \mathrm{~A}$, curve 1'). This is due to the removal of dissolved iron into the solution depth and easier formation of iron-ATMP complexes. The decrease in the concentration of the oxidized phase is also confirmed by the shift of the electrode potential to the negative region demonstrated in Figure 1 A.

It should be noted that a distinct pseudo-inductive loop is formed in the low-frequency region of the hodograph obtained in the stirred solution. It is known from literature that this loop is associated with electrochemical processes that occur via intermediate stages and with the existence of an adsorption equilibrium of the products of these reactions on the electrode surface [7]. For example, the following stages are possible in the system in question:

$$
\begin{gathered}
\mathrm{Fe}^{0} \rightarrow \mathrm{Fe}+(\mathrm{ad})+\mathrm{e} \\
\mathrm{Fe}^{+}(\mathrm{ad}) \rightarrow \mathrm{Fe}^{2+}+\mathrm{e}, \text { or } \mathrm{Fe}^{+}(\mathrm{ad})+\mathrm{OH}^{-} \rightarrow \mathrm{Fe}(\mathrm{OH})^{+}+\mathrm{e}
\end{gathered}
$$



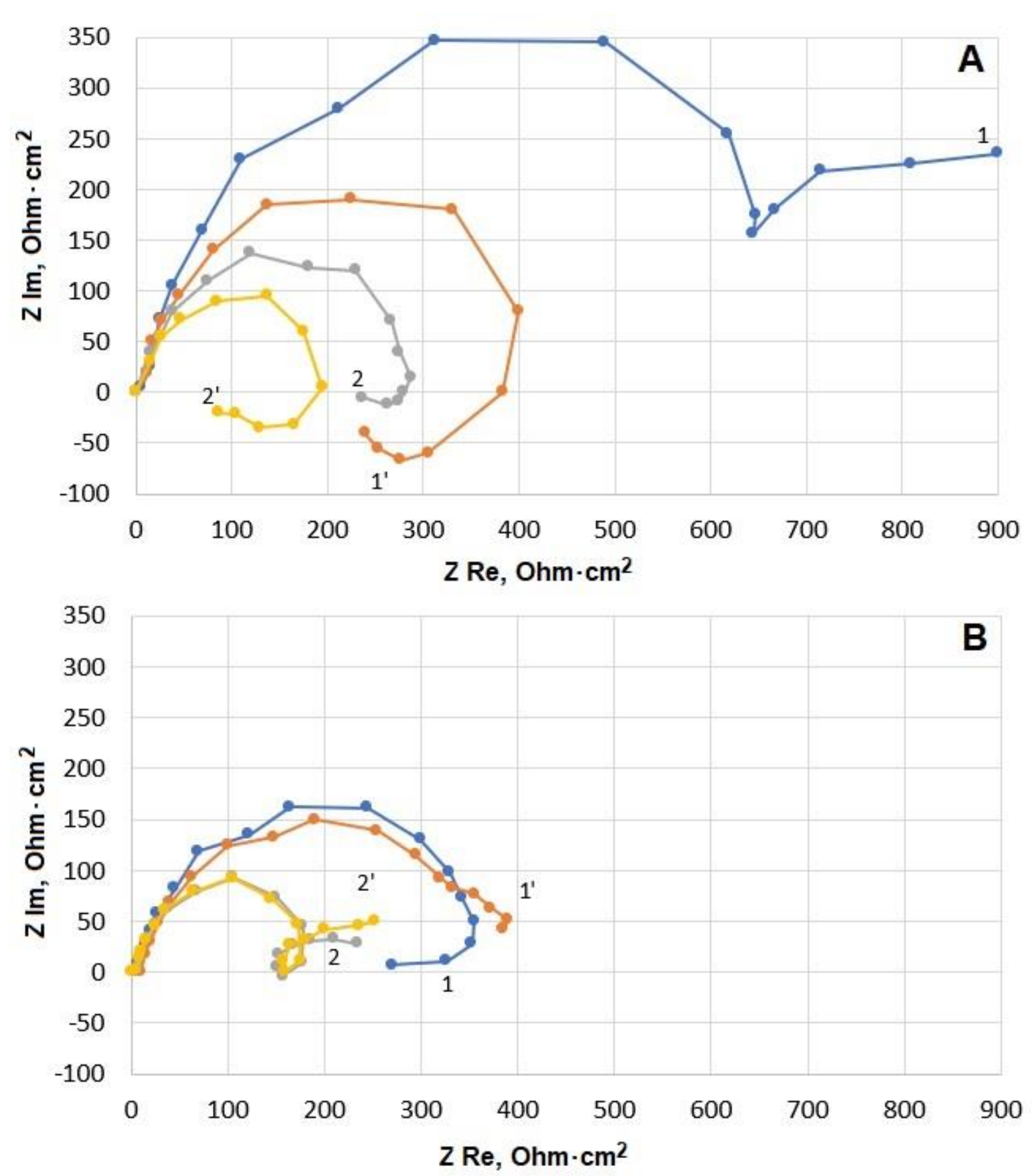

Figure 3. Nyquist plots. A - solution $1 ; \mathrm{B}-$ solution $2 ; 1,1^{\prime}-20^{\circ} \mathrm{C} ; 2,2^{\prime}-55^{\circ} \mathrm{C} ; 1,2-$ without stirring; $1^{\prime}, 2^{\prime}-$ with stirring.

With stirring, i.e., under conditions of intense removal of hydroxide ions formed by reaction (1) from the surface, the adsorbed intermediate corrosion products $\left(\mathrm{Fe}^{+}(\mathrm{ad})\right)$ do not form passivating hydroxide films, unlike in the unstirred solutions.

The nature of the hodographs obtained with heating (Figure 3A, curves 2,2') is completely identical to that of the hodograph obtained in the cold stirred solution, but heating 
the solution facilitates charge transfer and increases the corrosion rate of steel to a greater extent than stirring. Stirring the hot solution intensifies the described processes even more, as indicated by a decrease in the radius of the hodograph loop (Figure 3A, curve 2').

The conclusions drawn from an analysis of the Nyquist plots are confirmed by the values of corrosion currents determined from the corrosion plots of steel in an alkaline solution of ATMP (Figure 4).

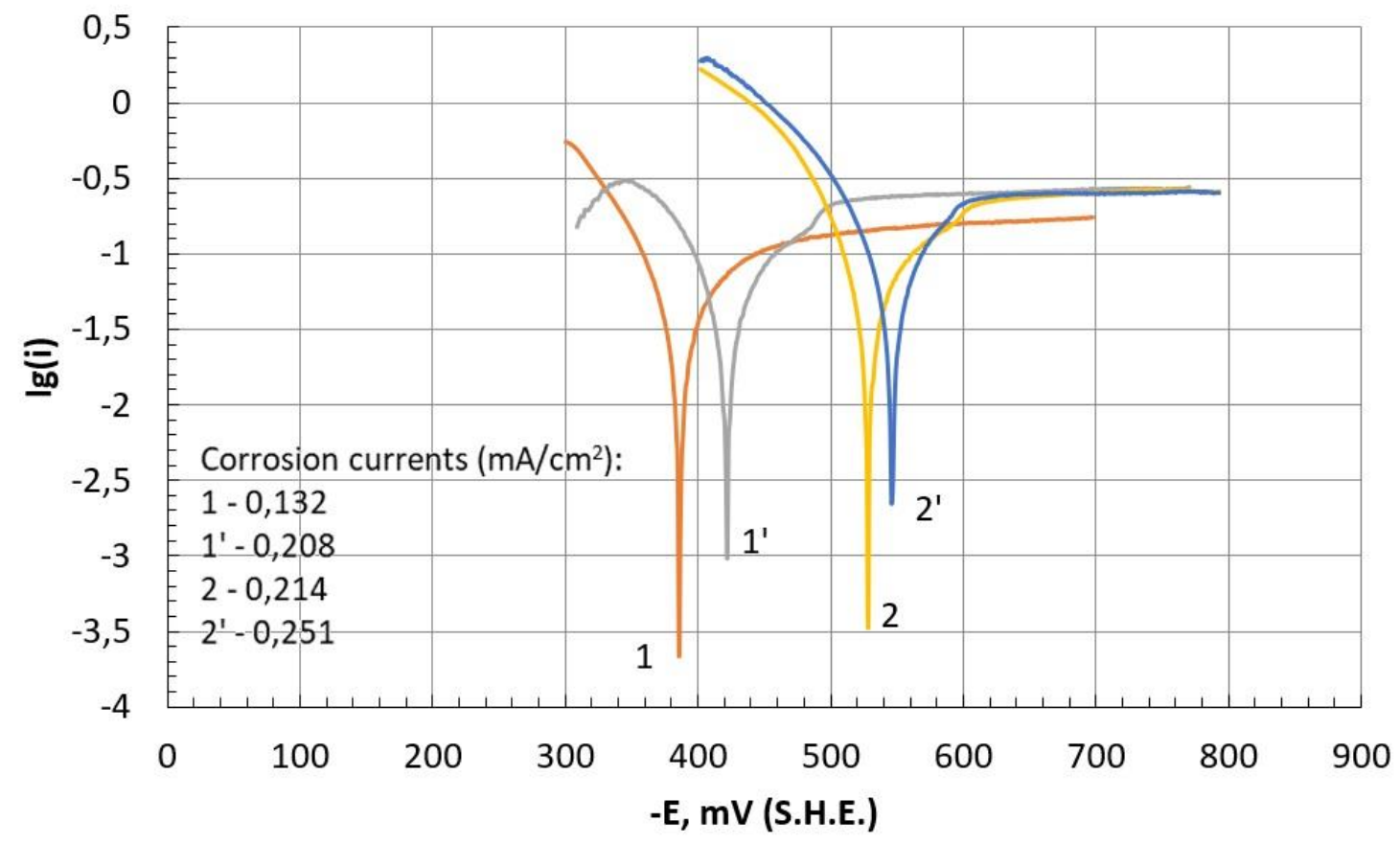

Figure 4. Corrosion plots of a steel electrode. Solution $1.1,1^{\prime}-20^{\circ} \mathrm{C} ; 2,2^{\prime}-55^{\circ} \mathrm{C} ; 1,2-$ without stirring; $1^{\prime}, 2^{\prime}-$ with stirring.

The addition of copper ions to the solution significantly affects the impedance hodographs (Figure 3B). First, the presence of copper in the solution significantly reduces the radius of the hodograph arc, which indicates that the corrosion processes in the system accelerate. This acceleration is more significant in the hot solution (Figure 3B, curves 2, 2'). It should be noted that in the presence of copper ions, stirring nearly ceases to have a noticeable effect on the hodographs. This fact can be explained by the absence of diffusion limitations on copper ions in the solution. The main differences in the behavior of the system at different temperatures in the presence of copper ions are observed in the low-frequency region. Pseudo-inductive loops are observed on the hodographs obtained in the cold solution (Figure 3B, curves $1,1^{\prime}$ ), indicating that adsorption layers are formed on the steel surface. In the hot solution, low-frequency loops are transformed to a so-called unresolved second semicircle, like in a cold solution without stirring (Figure 3A, curve 1), which may indicate that the nature of conductivity changes and stable passive films are formed on the electrode surface. 
The observed phenomena can be explained taking into account the concepts developed elsewhere $[6,8]$. According to these concepts, the following reactions can occur in the system being studied:

$$
\begin{gathered}
\mathrm{Fe}^{0} \rightarrow \mathrm{Fe}^{2+}+2 \mathrm{e} \\
{[\mathrm{CuHL}]^{n-}+\mathrm{Fe}^{2+} \rightarrow[\mathrm{FeHL}]^{n-}+\mathrm{Cu}^{2+}} \\
\mathrm{Fe}^{0} \rightarrow \mathrm{Fe}(\mathrm{OH})^{+}+\mathrm{e}^{-} \\
{[\mathrm{CuHL}]^{n-}+\mathrm{Fe}(\mathrm{OH})^{+} \rightarrow[\mathrm{Fe}(\mathrm{OH}) \mathrm{HL}]^{n-}+\mathrm{Cu}^{2+}} \\
\mathrm{Cu}^{2+}+2 \mathrm{OH}^{-} \rightarrow \mathrm{Cu}(\mathrm{OH})_{2} \downarrow \\
2 \mathrm{Cu}(\mathrm{OH})_{2}(\text { solid })+2 \mathrm{e}^{-} \rightarrow \mathrm{Cu}_{2} \mathrm{O}+\mathrm{H}_{2} \mathrm{O}+2 \mathrm{OH}^{-}
\end{gathered}
$$

The mechanism of formation of adsorption layers described by Equations (4)-(7) is supported by the results of XPS analysis of the surface layer of a steel sample kept for some time in solution 2 at $55^{\circ} \mathrm{C}$ that confirm the presence of mono- and bivalent copper compounds on the sample surface (Figure 5).
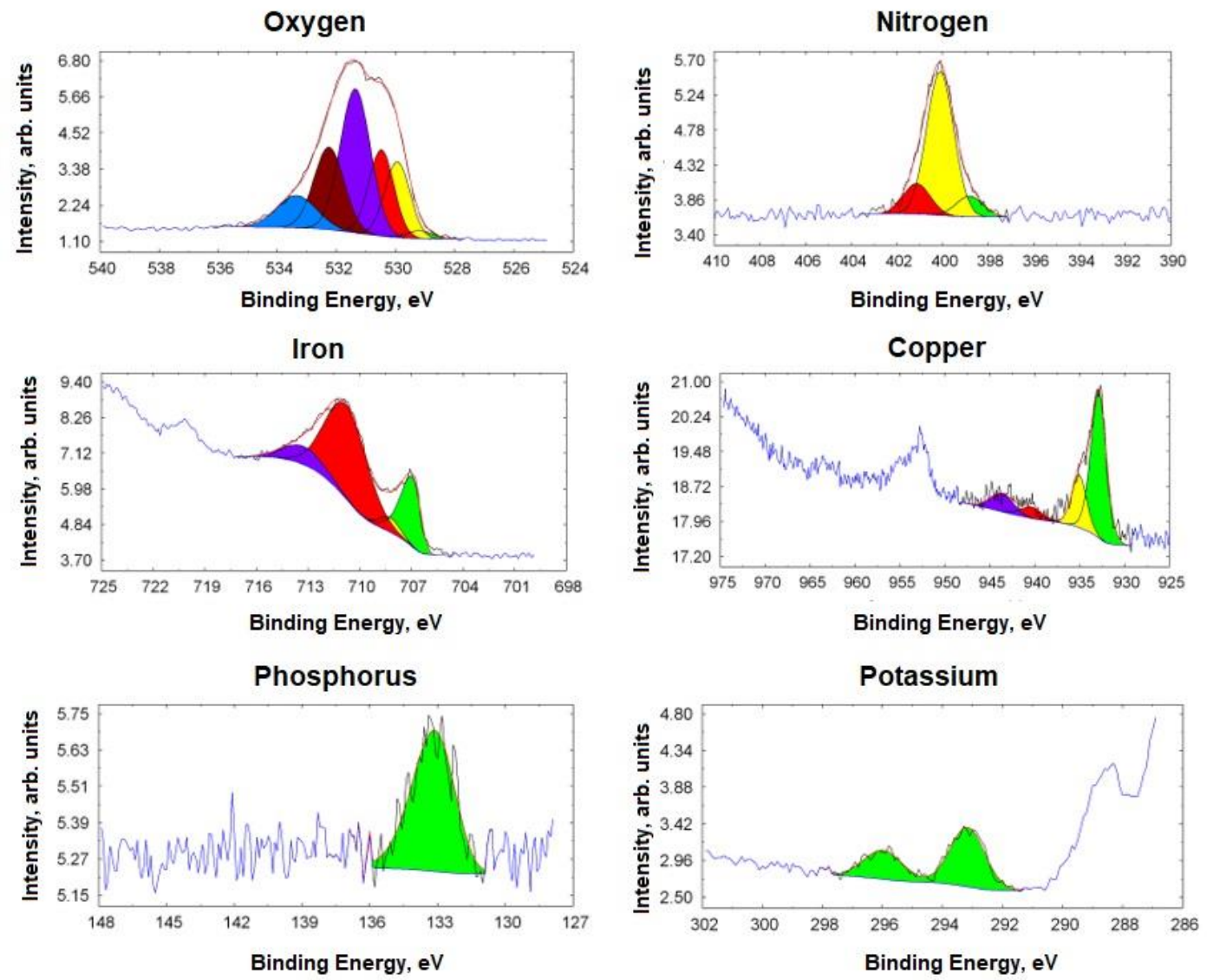

Figure 5. XPS spectra of the surface of a steel electrode. Solution 2, treatment time $15 \mathrm{~min}$, $t=55^{\circ} \mathrm{C}$, stirring. 
In addition to mono- and bivalent copper compounds, XPS spectra show that oxidized forms of iron, phosphorus, and potassium are present on the surface. This indicates that ligand molecules and iron dissolution products are also involved in the formation of passive films.

The results of elemental analysis are shown in Table 2.

Table 2. XPS analysis results.

\begin{tabular}{cc}
\hline Element & Content, at. \% \\
\hline Carbon & 62.19 \\
Oxygen & 27.43 \\
Nitrogen & 3.33 \\
Phosphorus & 0.77 \\
Iron & 4.08 \\
Copper & 1.48 \\
Potassium & 0.73 \\
\hline
\end{tabular}

\section{Conclusions}

1. It has been found that the inhibition of copper contact deposition is due to the formation of passivating adsorption films on the steel surface.

2. It has been shown that the formation of passivating layers is due to the electrophilic substitution of copper ions by iron ions formed upon steel corrosion in an ATMP solution.

3. It has been found that the passivating layers comprise compounds of mono- and divalent copper, iron, phosphorus, and potassium.

\section{Acknowledgements}

This study was supported by the Mendeleev University of Chemical Technology of Russia. Project Number X-2020-028.

\section{References}

1. Cl. Garhofer-Ondreicska and Ch. Garhöfer, Deposition of $\mathrm{Cu}, \mathrm{Sn}$, Zn-layers on metallic substrates, DE Patent WO2015039152 07.09.2015.

2. K. Bronder, S. Berger, B. Weyhmueller and U. Manz, Deposition of copper-tin and copper-tin-zinc alloys from an electrolyte, DE Patent WO2015/091201 Al 25.06.2015.

3. A.S. Pisarev, A.N. Serov, E.A. Zheludkova, A.A. Abrashov, N.S. Grigoryan, A.A. Kalinkina, E.A. Arkhipov and T.A. Vagramyan, Electrolyte for copper electrodeposition, RU Patent 2652328 25.04.2018 (in Russian).

4. F.F. Azhogin, M.A. Belen'kii, I.E. Gall' et.al., Gal'vanotekhnika, Metallurgiya, Moscow, 1987, pp. 175-177 (in Russian). 
5. V. Deluchat, J.-C. Bollinger, B. Serpaud and C. Caullet, Divalent cations speciation with three phosphonate ligands in the $\mathrm{pH}$-range of natural waters, Talanta, 1996, 44, 897907. doi: 10.1016/S0039-9140(96)02136-4

6. G.V. Zinchenko and Yu.I. Kuznetsov, Effect of oxidants on the protection of mild steel in water with zinc hydroxyethylidenediphosphonate, Zashch. Met., 2005, 41, 182-187 (in Russian).

7. V.V. Elkin, A.I. Marshakov, A.A. Rybkina and M.A. Maleeva, Interpretation of the impedance with negative capacitance and constant phase elements on iron electrode in weakly acid media, Elektrokhimiya, 2011, 47, no. 2, 147-158 (in Russian).

8. Y. Ru, H. Wei, Zh. Tianhua and M. Houyi, Corrosion protective performance of aminotrimethylene phosphonic acid-metal complex layers fabricated on the cold-rolled steel substrate via one-step assembly, Appl. Surf. Sci., 2018, 442, 264-274. doi: $\underline{10.1016 / j . a p s u s c .2018 .02 .167}$ 